\title{
Investigations on the Polarimetric Behavior of a Target near the Soil
}

\author{
Nicolas P. Marquart, Member, IEEE, Frédéric Molinet, Member, IEEE, and Eric Pottier, Senior Member, IEEE
}

\begin{abstract}
The polarimetric behavior of the diffracted field from an object located close to the ground is investigated for a varying incidence angle. Here, the field is described by the Geometrical Theory of Diffraction (GTD) in accordance to its asymptotic formulas $(\mathrm{k} \rightarrow \infty)$. As a result a ray system composed of 13 different rays was implemented for the monostatic case by applying the principle of Fermat. The different spatial and creeping waves give a physical insight in the mechanisms involved in the entire scattering process. By varying the angle from perpendicular to grazing incidence $0^{\circ}-90^{\circ}$, geometrical Surface Shadow Boundaries (SSB) are present for the backscattered field. At such boundaries the spatial waves are replaced by their corresponding creeping waves leading to a strong attenuation. The diffracted field for look angles related to the transition zones has a characteristic polarimetric behavior which can be represented on the Poincaré sphere. The typical locations on the sphere can be exploited in order to get information about the geometrical parameters of the target and its height above the ground.
\end{abstract}

Index Terms-Geometrical Theory of Diffraction (GTD), Uniform Theory of Diffraction (UTD), Surface Shadow Boundary (SSB), Polarimetry

\section{INTRODUCTION}

The diffraction of a plane wave by an object situated close to an interface of a two-layer medium has been investigated by many authors and an extensive literature exists on this topic [1], [2], [3] and [4]. However, only a few publications have been devoted to the analysis of the polarimetric behavior of the diffracted field. In general, very simple ray models based on the Geometrical Optic (GO) are considered involving single and double bounced waves for explaining some experimental results [5]. Here, this problem is again investigated by using a more refined ray model based on the Geometrical Theory of Diffraction (GTD). Depending on the geometrical properties of the target, lit and shadow regions arise in the backscattered GTD field for a variation from perpendicular to grazing incidence for a monostatic alignment of the transmitting and receiving antenna. The separating line of such a lit and shadow region is called a Surface Shadow Boundary (SSB). A special emphasis is attributed to the transition regions near the shadow boundaries where the reflected spatial waves disappear and transform into creeping waves. The creeping waves propagate on a arc length along the surface of the target. Approaching the shadow boundary the spatial waves are strongly attenuated and

Manuscript received February 15, 2006; revised March, 2006.

N. P. Marquart is with the German Aerospace Center (DLR) at the Microwave and Radar Institute, Oberpfaffenhofen, Germany.

F. Molinet is the director of the company MOTHESIM, La Boursidière, 92357 Le Plessis-Robinson Cedex, France. replaced by the powerless creeping waves. Hence, by reconsidering the problem according to the GTD not only the multiple reflections between the target and the ground of the spatial waves are investigated, but also the effects of creeping waves near the shadow boundaries on the polarimetric behavior of the target near the ground. Here, the refined ray system gives a better physical insight into the different mechanisms present of the entire scattering process.

\section{The RAy System AND ITS Singularities}

An important feature of a GTD ray field is based on the localization phenomenon, where the scattered field of an illuminated complex target can be viewed as the superposition of individual ray contributions from significant diffraction points (e.g. tips or edges). In consequence, these single points are locally replaced by canonical scatterers. For a smooth convex target in front of the air-soil interface the simplest model is given by a perfect conducting sphere or cylinder yielding the main features of the polarimetric behavior of any arbitrary convex target. Therefore, the backscattered field of a perfectly conducting cylinder located parallel above the ground is discussed in the following (fig. 1). An incident

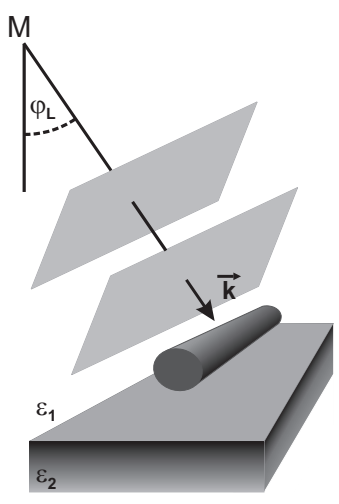

Fig. 1: GTD Model

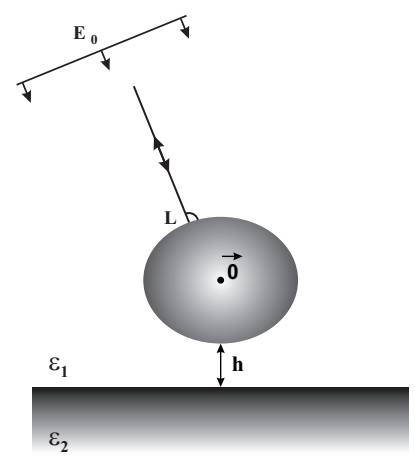

Fig. 2: Wave 1, height $h$ and phase origin monochromatic, unitary plane wave $\left|\vec{E}_{0}\right|=1$ radiated by an antenna located at far distance from the cylinder $(M)$ is considered. Here, the look angle $\varphi_{L}$ varies from $0^{\circ}$ to $90^{\circ}$. The distance between the bottom of the target and the soil is given by the height $h$. In the numerical calculation the phase origin $\overrightarrow{0}$ is set in the middle of the object (fig. 2). 13 waves of the ray system are found by applying Fermat's principle where the 
trajectories are straight lines as pointed out in the fig. 2- 14 . It can be seen from fig. 2 and fig. 8 that the specular wave 1 and the creeping wave 7 have no interaction with the soil. Due to the fact that a ray field is introduced, shadow boundaries occur for the field on its way back to the antenna. E.g. the waves 3 and 8 have a common shadow boundary where both are replaced by the creeping wave 4 (fig. 15(a)).

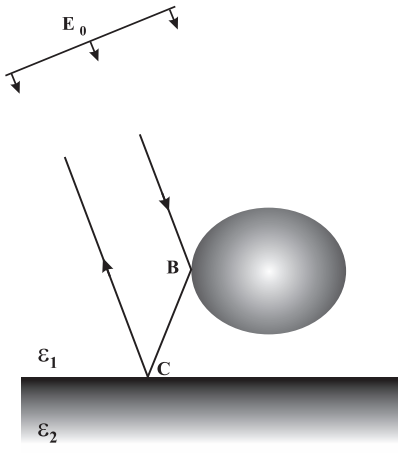

Fig. 3: Wave 2

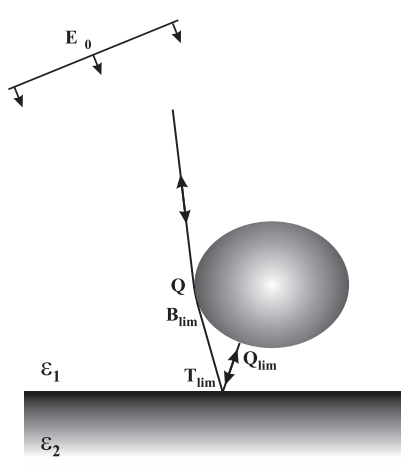

Fig. 5: Wave 4

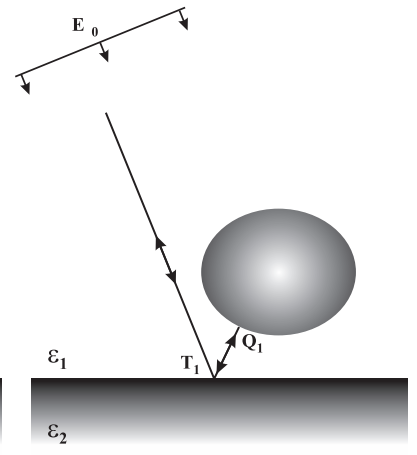

Fig. 4: Wave 3

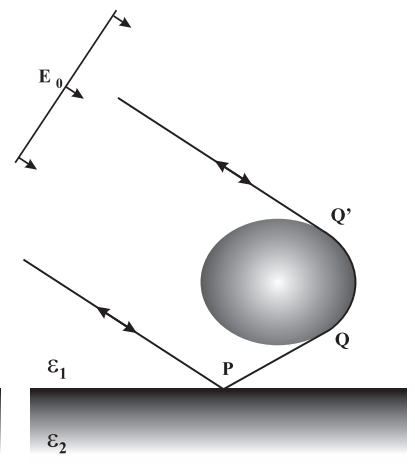

Fig. 6: Wave 5
A similar situation is on hand at the second shadow boundary related to wave 11 and 12 , both being substituted by wave 13 in the shadow region (fig. 15(b)). The correct field computation at a single transition from a lit into a shadow region is given by the Uniform Theory of Diffraction (UTD). But here, a sequence of two transition regions occurs, one on the way toward the ground and one on the way backward to the antenna. In fact, after a first interaction near grazing incidence on the cylinder the incident wave is transformed in a complex transition region field which is further reflected by the ground and the cylinder before a second interaction near grazing incidence on the way back to the receiving antenna. No analytical solution exists for the reflection of a transition region field by a curved convex surface or a planar non perfectly conducting surface. A way to treat this problem consists in decomposing the transition region field in a spectrum of plane waves and in applying to each spectral component the GO laws for the reflections on the interface and on the cylinder away from grazing incidence and the UTD for the second interaction at grazing incidence with the cylinder on the way back to the receiving antenna. Since this method is rather cumbersome to apply, a special procedure has been developed [6] to assume the continuity of the field across the sequence of surface shadow boundaries. This procedure which applies to waves 5, 6 and 9 will be presented in Section III. The waves 1 and 7 have no shadow boundaries, hence their interactions with the cylinder are defined after the GO and GTD. A similar treatment holds for waves 2 and 10 outside the transition region at $\varphi_{L}=0$. The transition region at $\varphi_{L}=0$ has not been considered in this analysis since for a 3-D object it overlaps with a caustic. In accordance to the asymptotic formulas the minimal height $h$ is constrained in the model to one wavelength $\left(h_{\min .} \approx \lambda\right)$ [7].

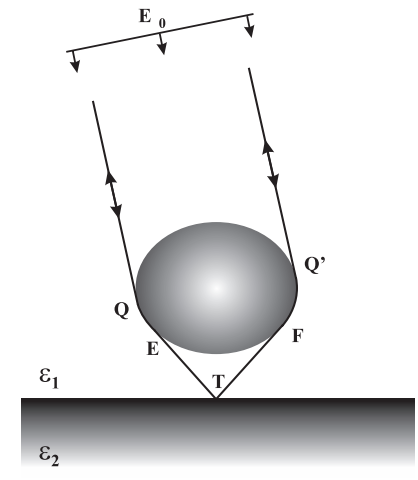

Fig. 7: Wave 6

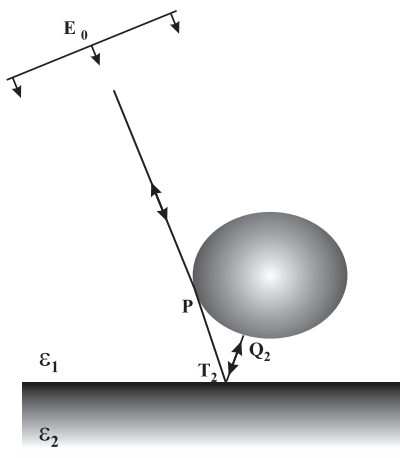

Fig. 9: Wave 8

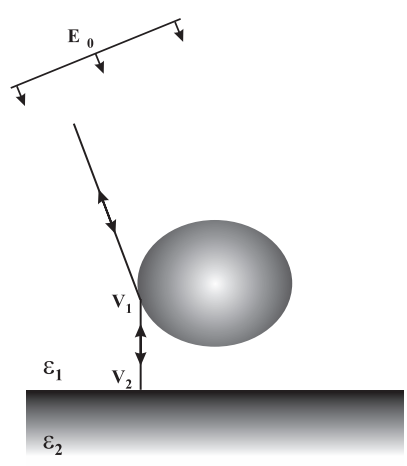

Fig. 11: Wave 10

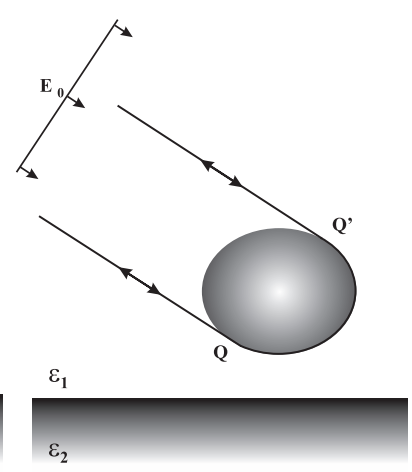

Fig. 8: Wave 7

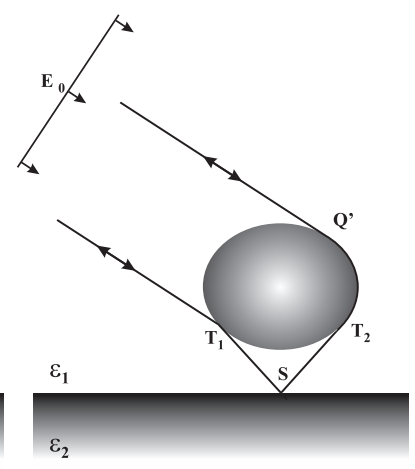

Fig. 10: Wave 9

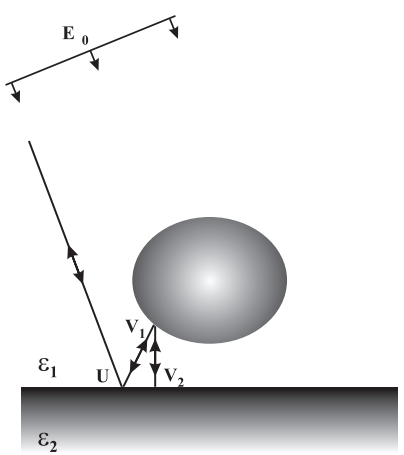

Fig. 12: Wave 11 


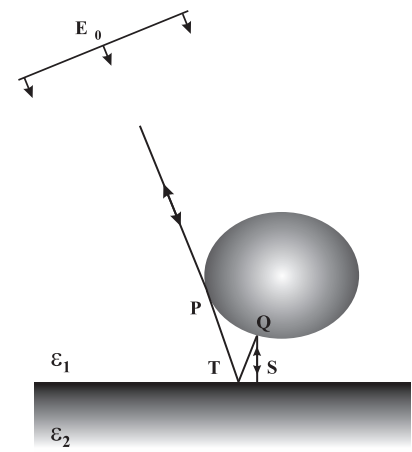

Fig. 13: Wave 12

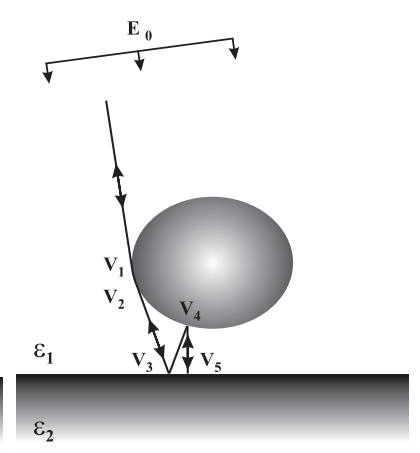

Fig. 14: Wave 13
A time-dependence $\exp (i \omega t)$ is considered and the phase origin is fixed in the middle of the cylinder. The divergence term $1 / \sqrt{0 M}$ and the round trip term $e^{-2 i \vec{k}_{1}^{i} \cdot \overrightarrow{0 M}}$ are extracted for every single wave for numerical purpose.

\section{TRANSITION ZONES}

In the ray system, the two shadow boundaries SSB 1 and SSB 2 are shown in fig. 15. Exemplarily, the field at the SSB1 is discussed in greater details in the following as the second boundary is similar. At the second boundary (SSB2) the waves 11 and 12 have simply one additional interaction with the cylinder and the interface. At the first boundary, corresponding to the look angle $\varphi_{S S B 1}$, the waves $3+8$ are strongly attenuated and finally vanish. Both waves are replaced in the shadowed region by the creeping wave 4 . In order to investigate the polarimetric behavior near these transition zones, the related fields must be calculated over the entire look angle range $0^{\circ}<\varphi_{L}<90^{\circ}$. Its calculation is discussed in the following and represents a cornerstone. Referring to Pathak [8] the UTD formulas are valid at any point in a single transition region for an incident local plane wave (GO-type field). Considering the two transition zones, the single field values $\vec{E}_{W 3}\left(Q_{1}\right), \vec{E}_{W 8}\left(Q_{2}\right), \vec{E}_{W 11}\left(V_{1}\right)$ and $\vec{E}_{W 12}(Q)$ up to the points $Q_{1}, Q_{2}, V_{1}$ and $V_{2}$ are therefore given accurately by the UTD. Here, at the transition the continuity of the total field is assured by the wave twosomes W3 + W8 and W11 $+\mathrm{W} 12$, where wave 3 and 11 correspond to the direct field and the wave 8 and 12 to the reflected field. A problem arises now in the calculation of the field on its way back to the antenna. As mentioned before, UTD does not apply to the reflection of a transition region field by a curved surface or a planar non perfectly conducting surface. The exact method to solve this problem would consist in a spectral decomposition of the transition region field in elementary plane waves. For each single elementary plane wave the UTD would again be applicable but this procedure is time-consuming. On the other hand the total field has to be continuous on the shadow boundary as in nature no jumps occur on shadow boundaries. This implies different conditions which base the Approximate Method presented next. The Approximate Method is based on the fact that the total field must be continuous at the transition from the lit into the shadow region.

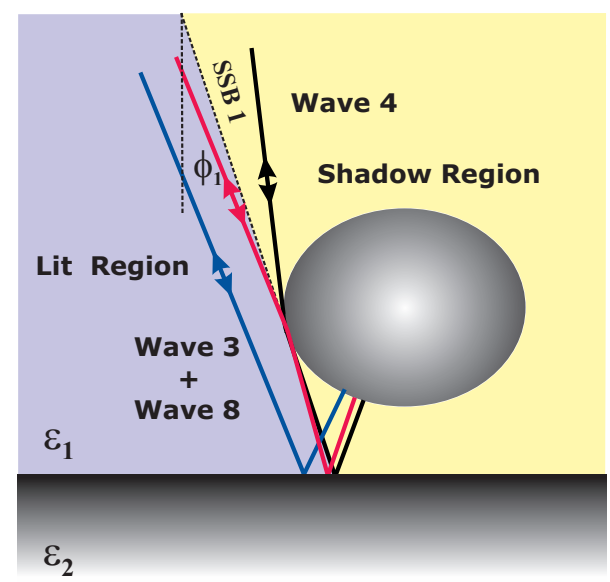

(a) SSB 1

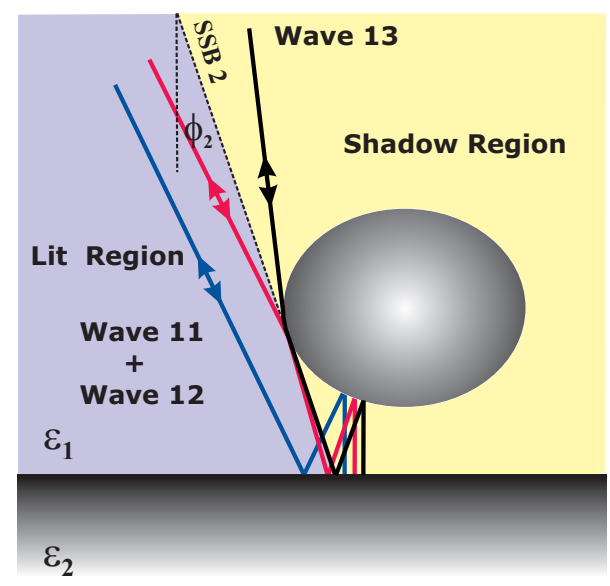

(b) SSB 2

Fig. 15: Transition Zones

This implies on the boundary:

$$
\begin{aligned}
\vec{E}_{3}^{i}(M)+\vec{E}_{8}^{r}(M) & =\vec{E}_{4}^{D}(M) \\
\vec{E}_{11}^{i}(M)+\vec{E}_{12}^{r}(M) & =\vec{E}_{13}^{D}(M)
\end{aligned}
$$

As the incident field $\vec{E}^{i}(M)$ is plane at grazing incidence, the relations (1), and (2) are only valid if the other two waves are also locally plane at the same time. This means that for the grazing incidence the Fourier components have a dominant contribution in the direction of grazing incidence $\left(\varphi_{S S B}\right)$. Therefrom, the incident waves $3+8$ at their reflection points $Q_{1}$ and $Q_{2}$ on the cylinder can be approximated by a plane wave when $Q_{1} \rightarrow Q_{2}$. Thus, both waves have the same radii of curvature after the reflection on 
the cylinder and the UTD formulas for the next diffraction at grazing incidence remain valid. The same applies for the waves 11 and 12 at their reflection points $V_{1}$ and $V_{2}$. Note that this method is only valid close to the two shadow boundaries. In the ray system the approximation was applied for the transition range $\varphi_{S S B}<\varphi_{L}<\varphi_{S S B}+5^{\circ}$. As a result of the Approximate Method the backscattered field over the whole look angle range $0^{\circ}<\varphi_{L}<90^{\circ}$ is subdivided into three segments: $\varphi_{S S B}+5^{\circ}<\varphi_{L}<90^{\circ}$ (Segment 1), $\varphi_{S S B}<\varphi_{L}<\varphi_{S S B}+5^{\circ}$ (Segment 2) and $0^{\circ}<\varphi_{L}<\varphi_{S S B}$ (Segment 3) which correspond to the lit, transition and shadow region. The principal shadow boundary (SSB 1) and its related segments are discussed exemplarily in this paper as the second shadow boundary (SSB 2) is computed in a similar manner. The general definition of the spatial divergence for a tube of rays is pointed out in [9] and [7]. The final divergence terms for the computation of the waves 3,4 and 8 are presented in detail in [6]. The calculation of the three field segments is discussed in the following.

\section{Lit region}

In a first step the waves 3 and 8 are calculated up to their reflection points $Q_{1}$ and $Q_{2}$ as outlined in fig. 4 and 9 after the UTD. Referring to fig. 4, it follows that no spatial divergence is given for wave 3 on its way to point $Q_{1}$ as at $T_{1}$ the flat interface has no influence on the radius of curvature. Wave 3 is just modified by the Fresnel coefficients $f$. Considering wave 8, it appears from fig. 9 that by approaching the shadow boundary, a grazing incidence $\left(\theta_{i}=\frac{\pi}{2}\right)$ is given at point $P$. Approaching the shadow boundary, the curvature at $P$ has the limit $1 / \rho_{2}(P) \rightarrow \infty$. Hence, the corresponding spreading factor $S F_{W}$ gets equal to zero. In order to get a continuous field at the transition, the uniform reflection dyad $\underline{R}^{a}$ must be applied. Assuming a unitary incident field $\vec{E}_{0}$ the UTD fields of the waves 3 and 8 at $Q_{1}$ and $Q_{2}$ are given by:

$$
\begin{aligned}
\vec{E}_{3,8}\left(Q_{1}, Q_{2}\right)= & \vec{E}_{0} \cdot \underline{f}\left(T_{1}\right) e^{-i\left[\vec{k}_{1}^{i} \cdot 0 \vec{T}_{1}+k_{1} T_{1} Q_{1}\right]} \\
+ & \vec{E}_{0} \cdot \underline{R}_{(P)}^{a} \cdot \underline{f}\left(T_{2}\right) S F_{W}\left(Q_{2}\right) \\
& e^{-i\left[\vec{k}_{1}^{i} \cdot 0 \vec{P}+k_{1}\left(P T_{2}+T_{2} Q_{2}\right)\right]}
\end{aligned}
$$

The first term refers to $\vec{E}_{3}\left(Q_{1}\right)$ and the second term describes the field $\vec{E}_{8}\left(Q_{2}\right)$. Where $\underline{f}\left(T_{1}\right)$ and $\underline{f}\left(T_{2}\right)$ are the Fresnel coefficients which describe the reflection on the ground. The spreading factor $S F_{W 8}\left(Q_{2}\right)$ defines the modified radius of curvature of the wave 8 after its interaction with the cylinder at point $P$. Considering the amplitudes $\vec{E}_{3}\left(Q_{1}\right)$ and $\vec{E}_{8}\left(Q_{2}\right)$ as new start values for calculating the field on its way back, it yields:

$$
\begin{gathered}
\vec{E}_{3,8}(M)=\vec{E}_{3}\left(Q_{1}\right) \cdot \underline{f}\left(T_{1}\right) S F\left(Q_{1}\right) e^{-i\left[\vec{k}_{1}^{r} \cdot 0 \vec{T}_{1}+k_{1} Q_{1} T_{1}\right]} \\
+\vec{E}_{8}\left(Q_{2}\right) \cdot \underline{f}\left(T_{2}\right) \cdot \underline{R}^{b}(P) S F(P) \\
\left.e^{-i\left[\vec{k}_{1}^{r} \cdot 0 \vec{P}\right.}+k_{1}\left(Q_{2} T_{2}+T_{2} P\right)\right]
\end{gathered}
$$

Where $\underline{R}_{(P)}^{b}$ and $S F(P)$ are here the reflection coefficient and spreading factor for way back of the round trip. Away from the boundary $\varphi_{S S B}+5^{\circ}<\varphi_{L}<90^{\circ}$ the summation of wave
3 and 8 is performed according to (4) and corresponds to a GTD field. In segment 1 the curvatures of the incident wave fronts of wave 3 and 8 at $Q_{1}$ and $Q_{2}$ are not considered as being plane. In the transition zone $\varphi_{S S B}<\varphi_{L}<\varphi_{S S B}+5^{\circ}$ the incident waves 3 and 8 at their reflection points $Q_{1}$ and $Q_{2}$ are considered as plane waves. Thus, both waves show approximately the same radius of curvature after their reflection and the UTD formalism can be applied.

\section{Boundary}

The exact value of the total field on the boundary is presented next. At grazing incidence the two points $Q_{1}$ and $Q_{2}$ coincide. The total field $\vec{E}_{38}^{S S B 1}$ at the reflection point $Q_{1}=Q_{2}$ on the boundary is given by [9]:

$$
\begin{aligned}
\vec{E}_{3,8}^{S S B 1}\left(Q_{1}=Q_{2}\right) & =\vec{E}_{0} \cdot\left[\underline{f}\left(T_{1}\right) e^{-i\left(\vec{k}_{1}^{i} \cdot 0 \vec{T}_{1}+k_{1} T_{1} Q_{1}\right)}\right. \\
& -\frac{1}{2} \underline{f}\left(T_{2}\right) e^{-i\left(\vec{k}_{1}^{i} \cdot 0 \vec{T}_{2}+k_{1} T_{2} Q\right)} \\
& -\underline{f}\left(T_{2}\right) m(P) \sqrt{\frac{2}{k_{1}}} e^{-i \frac{\pi}{4}} S F_{W 8}(Q) \\
& \left.e^{-i\left(\vec{k}_{1}^{i} \cdot 0 \vec{T}_{2}+k_{1} T_{2} Q\right)}\left\{\begin{array}{c}
p^{*}(0) \\
q^{*}(0)
\end{array}\right\}\right]
\end{aligned}
$$

Where $p^{*}$ and $q^{*}$ are the complex Fock scattering functions. The asterisk in (5) implies that the complex conjugate has to be taken in the case of a time-dependance $\exp (-i \omega t)$. After the reflection on the cylinder the backscattered field for grazing incidence is given by:

$$
\begin{aligned}
\vec{E}_{3,8}^{S S B}(M) & =\vec{E}_{3,8}\left(Q_{1}=Q_{2}\right) \cdot\left[\underline{f}\left(T_{1}\right) \cdot \underline{R}_{0}\left(Q_{1}\right) S F_{W 3}\left(Q_{1}\right)\right. \\
& e^{-i\left(\vec{k}_{1}^{r} 0 \vec{T}_{1}-k_{1} Q_{1} T_{1}\right)}-\frac{1}{2} \underline{f}\left(T_{2}\right) \cdot \underline{R}_{0}\left(Q_{2}\right) \\
& S F_{W 8}\left(Q_{2}\right) e^{-i\left(\vec{k}_{1}^{r} 0 \vec{T}_{2}-k_{1} Q_{2} T_{2}\right)}-m(P) \sqrt{\frac{2}{k_{1}}} \\
& \underline{R}_{0}\left(Q_{2}\right) \cdot \underline{f}\left(T_{2}\right) e^{-i \frac{\pi}{4}} S F_{W 8}(P) \\
& \left.e^{-i\left(\vec{k}_{1}^{r} 0 \vec{T}_{2}-k_{1} Q_{2} T_{2}\right)}\left\{\begin{array}{c}
p^{*}(0) \\
q^{*}(0)
\end{array}\right\}\right]
\end{aligned}
$$

\section{Shadow region}

In the shadow region the waves 3 and 8 are replaced by the creeping wave 4 . The latter creeps on a small arc length along the cylinder as shown in fig. 5 and leaves the surface tangentially at the detachment point $B$ again as a spatial wave toward the point $T$. The points $B, T$ and $F$ are fixed points and therefore must be computed only once. Here, only the attachment point $Q_{a t}$ varies on the cylinder. Referring to the uniform diffraction dyad $\underline{T}$ the field of wave 4 up to the point $Q$ is given as [9]:

$$
\begin{aligned}
\vec{E}_{4}(Q)= & \vec{E}_{0} \cdot \underline{T}^{a}\left(Q_{a t}, B\right) S F_{W 4}^{a}(Q) \\
& e^{-i\left[\vec{k}_{1}^{i} \cdot 0 \vec{Q}_{a t}+k_{1}(B T+T Q)\right]}
\end{aligned}
$$


The value $\vec{E}_{4}(Q)$ at $Q$ is taken as new input quantity for calculating the field on its way back. Therefrom, the received field at the antenna $M$ is computed as:

$$
\begin{aligned}
\vec{E}_{4(M)}= & \vec{E}_{4}(Q) \cdot \underline{T}^{b}\left(B, Q_{a t}\right) S F_{W 4}^{b}(B) \\
& e^{-i\left[-\vec{k}_{1}^{r} \cdot 0 \vec{Q}_{a t}+k_{1}(Q T+T B)\right]}
\end{aligned}
$$

In fig. 16 and 17 the different field calculations are presented for the real and imaginary parts of the parallel field component.

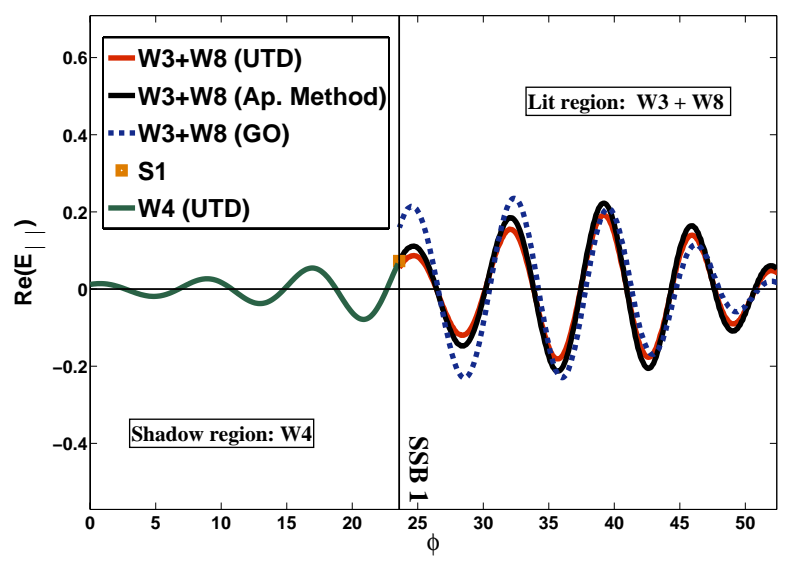

(a) Range $\phi$

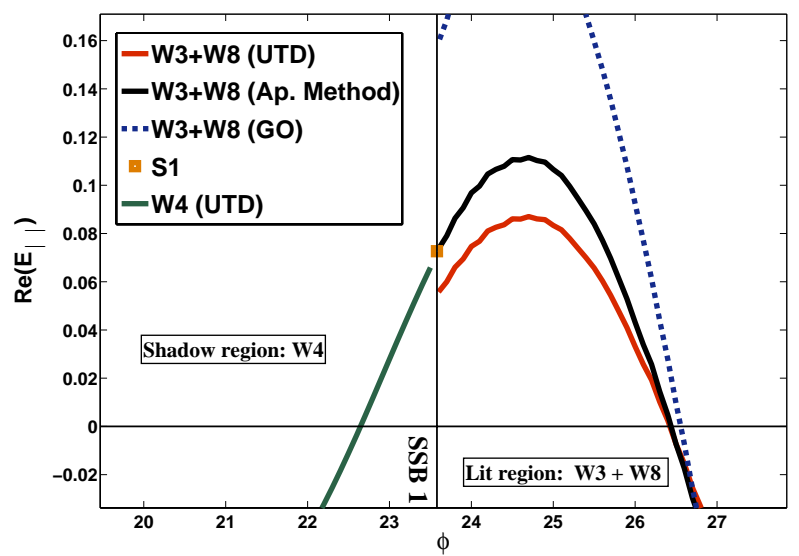

(b) Section SSB

Fig. 16: Real part of the $\vec{E}_{\|}$component. $\left[\vec{E}_{\|}^{0}=1, \vec{E}_{\perp}^{0}=1, f=500 M H z, \epsilon_{2}=9.6\right]$

Here the GO abbreviation (e.g. fig. 16) of the field denotes that the reflection on the cylinder is given over the entire look angle range by \pm 1 corresponding to the parallel and perpendicular field components. The points $S_{1}$ and $S_{2}$ are the exact field values on the shadow boundaries. It can be

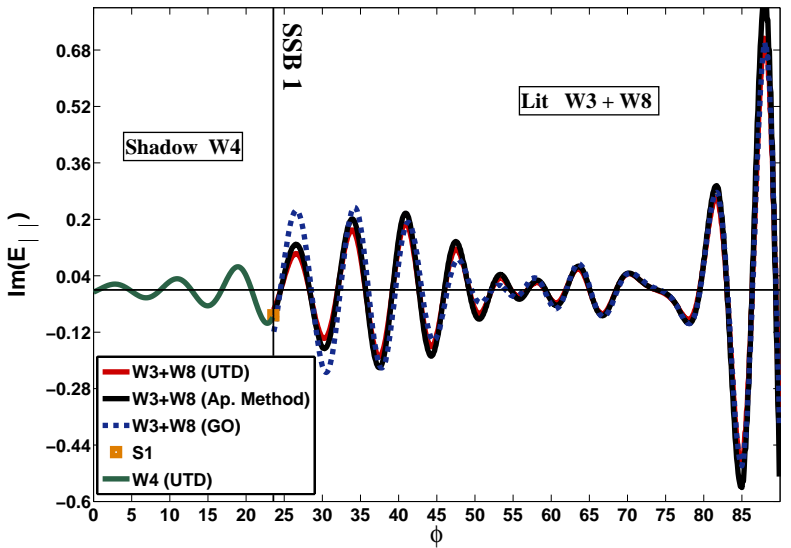

(a) Range $\phi$

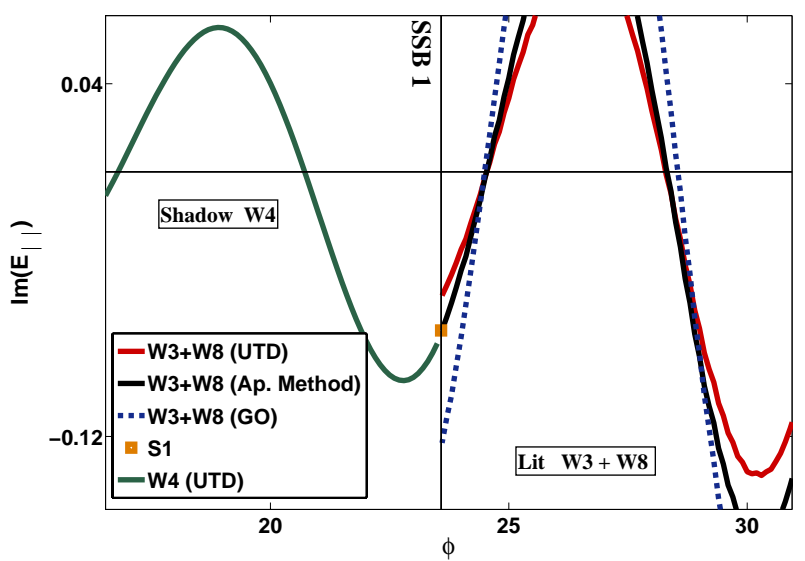

(b) Section SSB

Fig. 17: Imaginary part of the $\vec{E}_{\|}$component. $\left[\vec{E}_{\|}^{0}=1, \vec{E}_{\perp}^{0}=1, f=500 M H z, \epsilon_{2}=9.6\right]$

seen that away from the transition zones the UTD and the GO fields are equal. The combination of the three field segments was performed by a polynomial fit. Similar results are obtained for the imaginary part. In the same way a polynomial fit was performed for the waves 11,12 and 13 related to the second transition zone (SSB 2). Thus, a continuous ray field is given. The final wave contributions according to the Approximate Method is shown in fig. 18(a) and 18(b) for a dielectric soil $\epsilon_{2}=9.6$. At the corresponding shadow boundaries the related spatial waves are strongly attenuated. Further, for the parallel field component the Brewster angle is given at $72.1^{\circ}$. The introduce Approximate Method represents an innovation in the general UTD field calculation. Here the field can be calculated accurately at the transition from a lit into a shadow region due to a geometrical surface shadow boundary. 


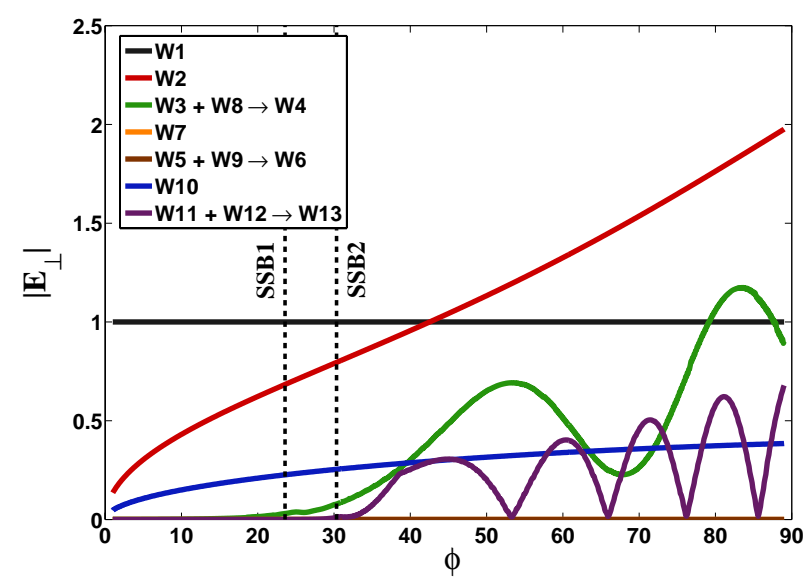

(a) $E_{\perp}$

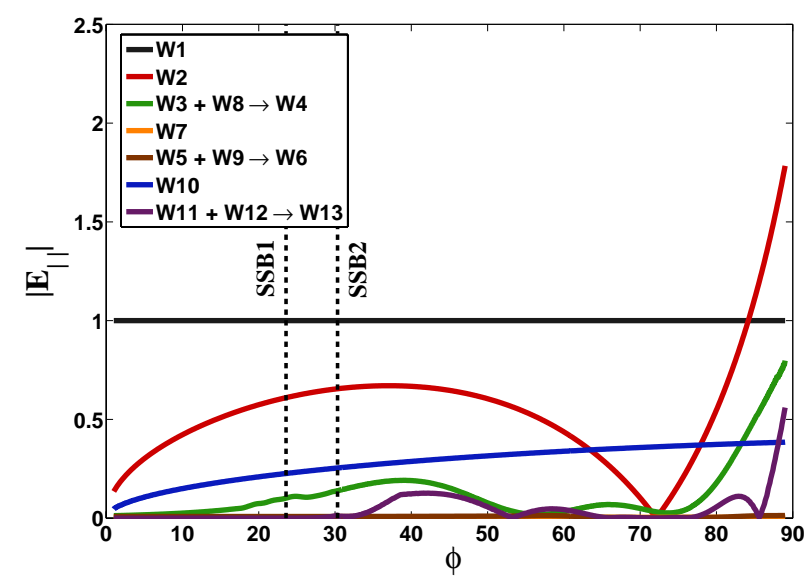

(b) $E_{\|}$

Fig. 18: Single wave contributions

\section{NUMERICAL VALIDATION}

As the contribution of the considered bundle of rays is at the end an approximation of the total field, the accuracy of the field obtained with the implemented ray system has to be checked. This was done by an exact numerical integral full wave solution solved via the Method of Moments (MoM) [6]. Here, the effect of the soil was introduced by a modified Green dyad for a two-layer media, where according to Michalski's method [4] the Electric Field Integral Equation (EFIE) was transformed in the less singular Mixed Potential Integral Equation (MPIE). The related Sommerfeld integrals in the extended MoM were calculated after the Discrete Complex Image Method (DCIM) [10]. The validation was performed for a perfect conducting sphere situated above a dielectric soil. Where the diffracted field was calculated once for a soil with permittivity $\epsilon_{2}=9.6$ and once for the double value 19.2. The waves $1,2,3,10$ and 11 were determined according to the GTD model described before and adapted for the sphere. In comparison to the cylinder, the corresponding spreading factors were modified as here the perpendicular radius of curvature relative to the plane of incidence is not infinite $\left(\rho_{1} \neq \infty\right)$. The waves 3 and 11 were computed in the lit region up to their shadow boundaries. The Radar Cross Section (RCS) of both numerical techniques for the $E_{\| \mid}(M)$ and $E_{\perp}(M)$ components are presented in fig. 19 and fig. 20. A good agreement between the asymptotic field description of the GTD and the adapted MoM has been obtained in the range $8^{\circ}<\varphi_{L}<90^{\circ}$. According to the GTD field description Wave 2 and 10 have a transition region for the vertical incidence $\varphi_{L}=0$. In our analysis, the shadow boundary transition region of waves 2 and 10 at $\varphi_{L}=0$ will not be treated since, in the case of a three-dimensional object, this direction is also a caustic direction for these waves and needs therefore a different approach. Hence, the GTD result brakes down for the perpendicular incidence as outlined in fig. 19 and 20. Here, this special incidence is not of interest as the polarimetric behavior near the transition zones is investigated. Compared to the computing based on the MOM technique which is very time consuming, the introduced Approximate Method in accordance to the GTD calculation slashes down CPU time significantly. Moreover, the refined GTD ray system gives a better physical insight into the different scattering mechanisms in comparison to a full wave solution like the MoM. In consequence, the geometrical description of the scattering problem and the related transition zones can now be exploited in order to get information about the geometrical properties of the set-up.

\section{Representation on the Poincaré sphere}

The introduced ray system is considered in the following to describe the polarization of the backscattered field from a cylinder located near the soil (fig. 1). Here, the polarization of the field is represented in a first step on the Poincare sphere in order to outline the polarimetric behavior of the diffracted field for incidence angles close to the geometrical shadow boundaries. Therefore, the Poincare sphere is briefly introduced next. The backscattered field measured at the antenna is described by the Jones vector as follows:

$$
\vec{E}=\left[\begin{array}{ll}
\left|E_{\perp}\right| & e^{i \Phi_{\perp}} \\
\mid E_{\|} & e^{i \Phi_{\|}}
\end{array}\right]
$$

Based on power measurements, Stokes introduced a power vector in order to describe partially polarized waves. Refer to (9) the Stokes vector $\vec{g}$ is defined straightforward by means of the two field component as follows:

$$
\vec{g}=\left[\begin{array}{c}
g_{0}=\left|E_{\perp}\right|^{2}+\left|E_{\|}\right|^{2} \\
g_{1}=\left|E_{\perp}\right|^{2}-\left|E_{\|}\right|^{2} \\
g_{2}=2 \operatorname{Re}\left(E_{\perp} E_{\|}^{*}\right) \\
g_{3}=-2 \operatorname{Im}\left(E_{\perp} E_{\|}^{*}\right)
\end{array}\right]
$$




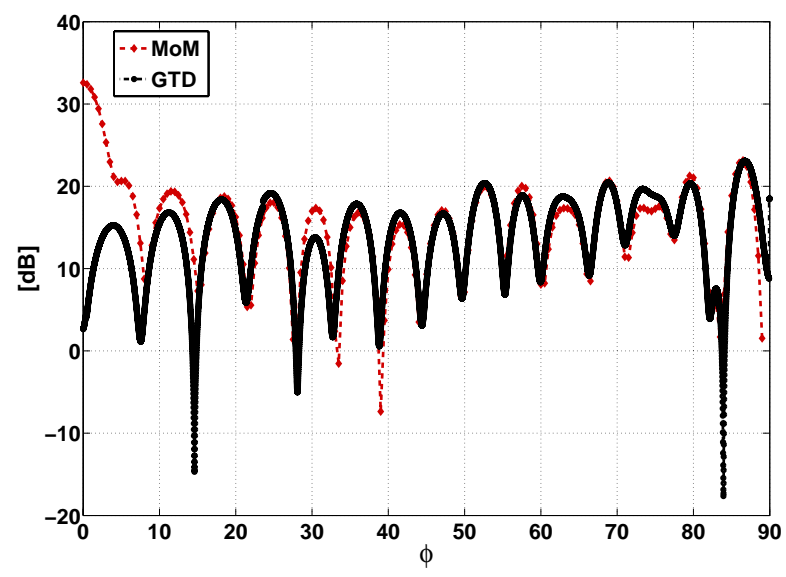

(a) $E_{\perp}$

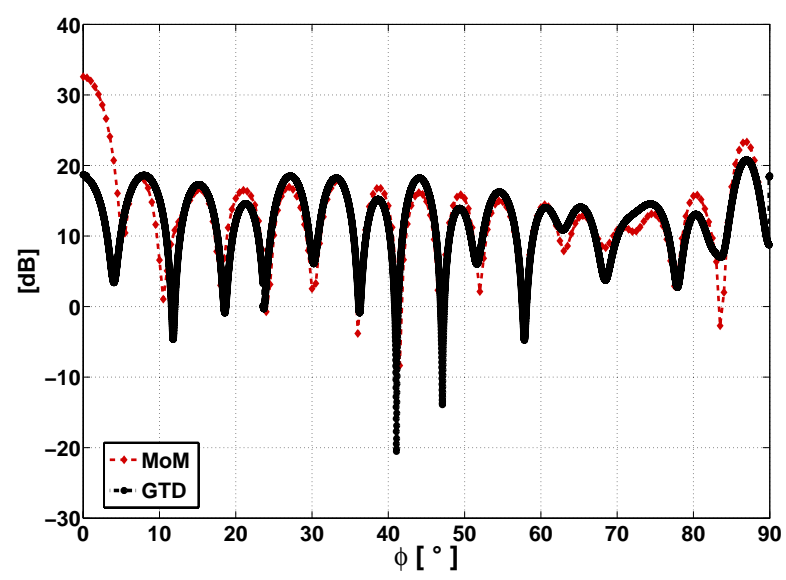

(b) $E_{\|}$

Fig. 19: RCS sphere after the GTD and MoM

$\left[k=10, r=2, h=0.5, \epsilon_{2}=9.6\right]$

Where the component $g_{0}$ expresses the total field intensity. In the case of a total polarized field, it yields:

$$
g_{0}=\sqrt{g_{1}^{2}+g_{2}^{2}+g_{3}^{2}}
$$

The components $g_{1}$ and $g_{2}$ describe the linear polarization. The $g_{3}$ component points out either a left or right handed circulation of the diffracted field. The Stokes component $g_{1} g_{2}$ and $g_{3}$ in (10) can be regarded as the spherical coordinates of a sphere with the radius $g_{0}$. Hence, considering a normalized sphere $g_{0}=1$ the different polarization states related to the different incidence angles can be represented on a common single spherical surface. According to the IEEE definition (Std. 211-1997) the upper hemisphere of the sphere describes here a left handed polarized field and the upper pole signifies

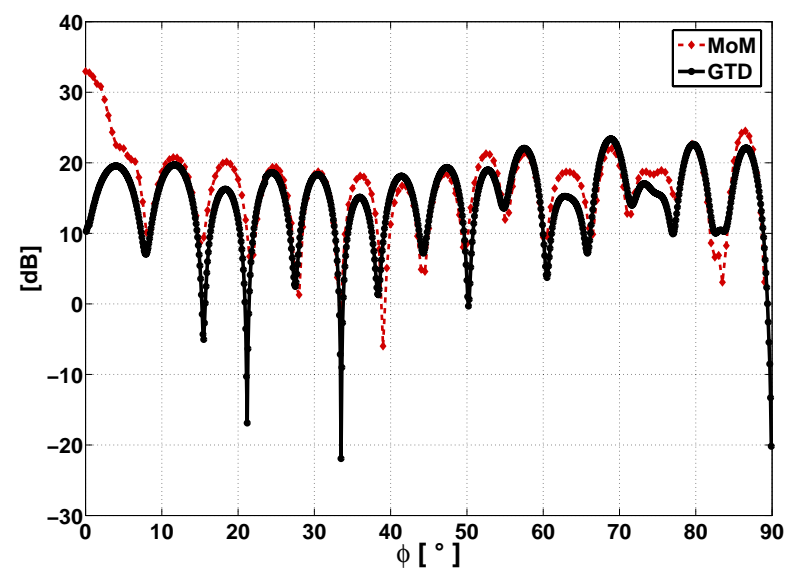

(a) $E_{\perp}$

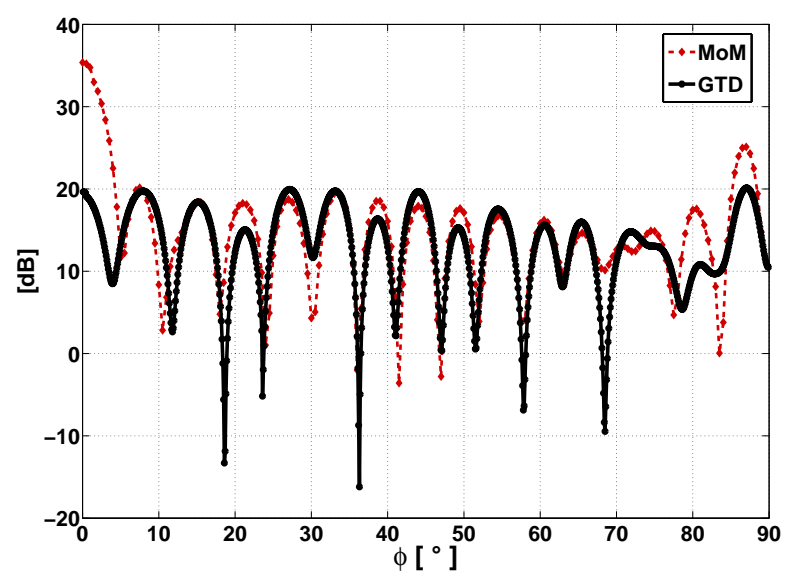

(b) $E_{\|}$

Fig. 20: RCS sphere after the GTD and MoM $\left[k=10, r=2, h=0.5, \epsilon_{2}=19.2\right]$

a Left Handed Circular (LHC) polarization state. In contrast the bottom hemisphere describes a right handed polarization and the pole a Right Handed Circular (RHC) polarization. The equator line characterizes a linear polarization state where $g_{2}=g_{3}=0$. In fig. 21 , the polarization states of the received field is represented on the Poincaré sphere for a linear incident polarized field over the whole look angle range $0^{\circ}-90^{\circ}$. The diffracted field was computed for an angle increment $\Delta$ of $0.1^{\circ}$. In fig. 21(a) and 21(b) the polarization of the received field in accordance to the GTD model (fig. 1) is outlined for a linear polarized incidence field $E_{\perp}=E_{\|}=1$ once for a perfect conducting interface and once for a dielectric soil $\epsilon_{2}=9.6$. Referring to fig. 21(b) the scattered field at perpendicular incidence $\varphi_{L}=0^{\circ}$ is located near $g_{2}=1$ in accordance to the Stokes vector (10). With increasing look angle the different ray paths in the system and the corresponding phase terms vary. As a result, 
the superposition of the different complex rays yield to a rapid variation of the Sinclair matrix $[S]$ over the whole look angle range. This is noticed as a rapid cycling on the Poincaré sphere. This behavior is pointed out in fig. 21(a) and fig. 21(b). In the case where a perfect conducting interface is given the Fresnel coefficients at the interface are constantly given by $\underline{f}=\hat{e}_{\|}-\hat{e}_{\perp}$. Hence, the change in the path lengths leads to a strong cycling on the sphere. Close to the shadow

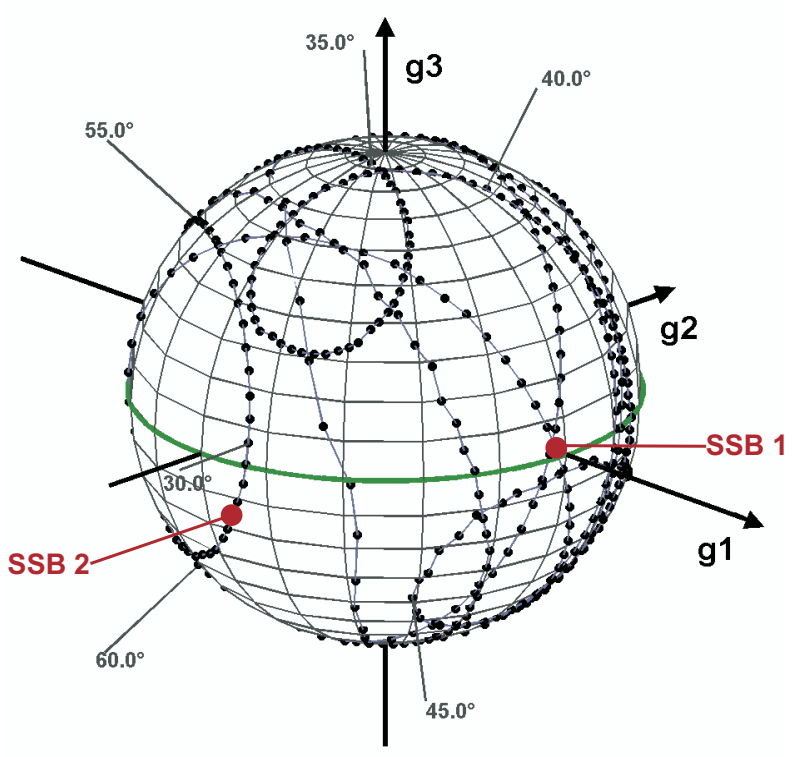

(a) Perfect Conducting Interface

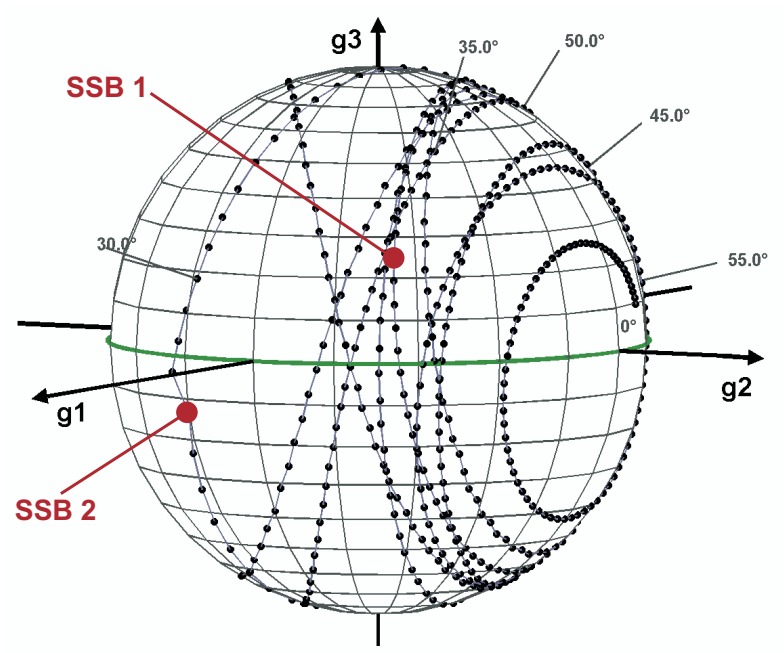

(b) Dielectric Soil $\left(\epsilon_{2}=9.6\right)$

Fig. 21: Linear polarized incident field

boundaries the related spatial waves have similar path lengths thus being in phase. This lead to a slighter depolarization of the backscattered field. Note that the direct reflected wave 1 from the object depends not of the incidence angle. The wave 1 has a constant value over the entire range (fig. 18(a) and 18(b)). The spatial waves related to the shadow boundaries are strongly attenuated in the transition regions being finally replaced by the corresponding creeping wave in the shadow region. Hence, the polarization of the received field for incidence angles close to the shadow boundaries are found near the equator line representing a linear polarization state such as the transmitted field.

\section{CONCLUSION}

A GTD ray system composed of 13 different waves including spatial and creeping waves was numerically implemented for a varying incidence angle. The ray system was validated by an exact numerical method. In this paper it was shown that the considered ray contributors describe the field accurately. Using the ray system, a better physical insight is given in the entire scattering process. For a varying incidence angle from the perpendicular to grazing case, so-called Surface Shadow Boundaries occur for the diffracted ray field, where the spatial waves disappear and transform into creeping waves. The corresponding look angles have characteristic locations on the Poincaré sphere. Considering e.g. a linear polarized incidence field the transition zones have characteristic locations close to the equator line signifying a linear polarization. Hence, the depolarization close to the shadow boundaries is less and similar to the incident field. In consequence these characteristic locations can be exploited to determine the size of a cylinder or sphere and its height above the ground. Furthermore, due to the fact that the GTD implies the localization phenomenon any convex curved object can be treated in this manner. In this case the characteristic diffraction points of the complex object are considered.

\section{ACKNOWLEDGMENT}

The authors would like to thank Stephane Tort from the company MOTHESIM for his help in implementing the modified MPIE.

\section{REFERENCES}

[1] C. M. Butler, X. Xiao-Bang, and A. Glisson, "Current induced on a conducting cylinder located near the planar interface between two semiinfinite half-spaces," IEEE Transactions on Antennas and Propagation, vol. 33, pp. 616-624, Mar. 1990.

[2] Q. Chen and D. R. Wilton, "Electromagnetic scattering by threedimensional arbitrary complex/conducting bodies," Antennas and Propagation Society International Symposium, vol. 2, pp. 590-593, May 1990.

[3] N. Geng, "Fast multipole method for scattering from an arbitrary pec target above or buried in a lossy half space," IEEE Transactions on Antennas and Propagation, vol. 49, pp. 740-748, May 2001.

[4] K. A. Michalski and D. Zheng, "Electromagnetic scattering and radiation by surfaces of arbitrary shape in layered media, part i: Theory," IEEE Transactions on Antennas and Propagation, vol. 38, pp. 335-344, Mar. 1990.

[5] E. Krogager, Aspects of Polarimetric Radar Imaging. Danish Defence Research Establishment, 1993.

[6] N. P. Marquart, "Investigation on the polarimetric behavior of the em-field scattered by an object located near the interface between the air and a lossy dielectric half-space," Ph.D. dissertation, Univ. of Rennes 1, Institute of Electronique and Telecommunications of Rennes, 263, av. du Générale Leclerc, 35042 Rennes Cedex, France, Jan. 2006. [Online]. Available: http://tel.ccsd.cnrs.fr

[7] D. Bouche, F. Molinet, and R. Mittra, Asymptotic Methods in Electromagnetics. Berlin, Heidelberg and New York: Springer-Verlag, 1997. 
[8] P. M. Pathak, "An asymptotic analysis of the scattering of plane waves by a smooth convex cylinder," Radio Science, vol. 14, no. 3, pp. 419435, May 1979.

[9] D. A. McNamara, C. W. I. Pistorius, and J. A. G. Malherbe, Indroduction to the Uniform Geometrical Theory of Diffraction. Boston and London: Artech House, 1990.

[10] F.Molinet and S.Tort, "Modelisation d'un reseau fin large bande," MOTHESIM, Rapport final d'etude, Marche $N^{o} 085 / 714 / C N E S / 97 / 6766 / 00$ Document MOTHESIM $N^{o} 609$, Mar. 1999.

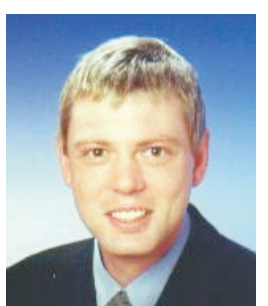

Nicolas Pascal Marquart (M'06) was born in Ehingen (Donau), Germany, on April 26, 1971. He received the degree Dipl.-Geophys. in 2000 from the University of Karlsruhe, Germany in the area Ground Penetrating Radar. He received the Ph.D. degree in 2006 from the University of Rennes 1, France in the work area electromagnetic diffraction theory and Radar polarimetry.

From 2000 to 2003 he worked at the company MOTHESIM, France in the frame of the Training and Mobility program for young Researchers (TMR) of the European Commission. From 2004-2005 he joined the Research Training Network (RTN) of the European Commission and worked in the project: Application of Multiparameter Polarimetry in Environmental Remote sensing (AMPER) at the Institute for the Protection and Security of the Citizen at the Joint Research Centre in Ispra, Italy. He is currently working at the Microwaves and Radar Institute (HR) at the German Aerospace Center (DLR). His current activities of research are centered on the Tsunami-Early-WarningSystem (TEWS) project and Dual pol calibration in the ALOS PALSAR Products Verification project.

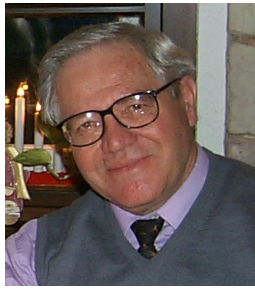

Frédéric Molinet (M'82) was born in Wengelsbach, France on November 13, 1934. He received the Engineer degree from the Ecole Centrale de Lyon in 1959, the Doctor de spécialité ( $3^{e}$ cylcle) in theoretical nuclear physics from the University of Strasbourg and the Doctor-és-Sciences degree from the University of Paris in 1971.

From 1964 to 1971, he was involved in research in plasma physics at the Institut Henri Poincaré During 1971, he joined the Laboratoire Central de Télécommunications as a group leader of the Department of Theoretical Studies and Modeling. In 1980, he founded MOTHESIM, where he has been active in the development of numerical and asymptotic techniques for the electromagnetic and acoustic radiation and scattering problems, with applications to EMP, EMC Radar and Sonar scattering. Dr. F. Molinet is a former president of the Waves and Fields chapter of the Sociéte des Electrisiens, Electroniciens et des Radioélectriciens (SEE) and the chairman of the French URSI Commission B.

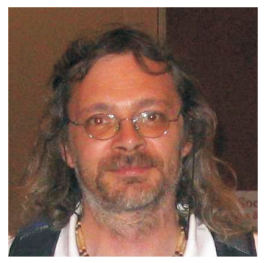

Eric Pottier (M'95, SM'06) received the MSc and Ph.D. in signal processing and telecommunication from the University of Rennes 1, respectively in 1987 and 1990, and the Habilitation from the University of Nantes in 1998. From 1988 to 1999 he was an Associate Professor at IRESTE -University of Nantes, Nantes, France where he was the Head of the Polarimetry Group of the Electronic and Informatic Systems laboratory. Since 1999, he has been a Full Professor at the University of Rennes 1, France, where he is currently the Deputy Director of the Institute of Electronics and Telecommunications of Rennes (I.E.T.R - CNRS UMR 6164) and also Head of the Image and Remote Sensing Group - SAPHIR Team. His current activities of research and education are centered in the topics of analog electronics, microwave theory and radar imaging with emphasis in radar polarimetry. His research covers a wide spectrum of areas from radar image processing (SAR, ISAR), polarimetric scattering modeling, supervised/unsupervised polarimetric segmentation and classification to fundamentals and basic theory of polarimetry. Since 1989, he has supervised more than 50 research students to graduation (MSc and $\mathrm{PhD}$ ) in Radar Polarimetry covering areas from theory to remote sensing applications. $\mathrm{He}$ has chaired and organized 29 sessions in International Conferences and was member of the Technical and Scientific Committees of 17 International Symposium or Conferences. He has been invited to present 30 presentations in International Conferences and 15 in National Conferences. He has 7 publications in books, 38 papers in refereed journals and more than 220 papers in Conference and Symposium proceedings. He has presented advances courses and seminars on Radar Polarimetry to a wide range of organization and events (DLR, NASDA, JRC, RESTEC, ISAP2000, IGARSS03, EUSAR04, NATO04, PolInSAR05, IGARSS05, JAXA06, EUSAR06, NATO-06). He received the Best Paper Award during EUSAR2000 for his research activities, coauthored with J.S. Lee (U.S. Naval Research Laboratory), in the topic of polarimetric unsupervised segmentation of POL-SAR data. 